\title{
Research on subjective evaluation method of automatic emergency braking (AEB) for passenger car
}

\author{
Shuai Zhang*, Bin Lei, Shuai Chen, and Lei Sha \\ CATARC Automotive Test Center(Tianjin) Co.,Ltd., Tianjin 300300, China
}

\begin{abstract}
Keywords: passenger car, automatic emergency braking, subjective evaluation.

Abstract. With the continuous development of automotive active safety technology, automatic emergency braking technology has been widely used, playing an important role in avoiding rear-end collisions or collisions with pedestrians and other traffic participants. This article analyzes the technical characteristics of the automatic emergency braking system, put forward subjective evaluation index, select vehicle-to-vehicle, vehicle -to-pedestrian and other typical scenes for automatic emergency braking subjective evaluation and actual vehicle verification, and constructed a subjective evaluation system for automatic emergency braking of passenger car.
\end{abstract}

\section{Foreword}

Automotive safety technology is divided into passive safety technology and active safety technology. In the early stages of the development of automotive safety technology, limited by technical conditions, passive safety technology is mainly considered in automotive safety design, like high-strength car body, bumper, seat belt, safety headrest, airbag, etc. The main function of passive safety technology is to reduce the personal injury to the occupants of the car after a car accident, while traditional passive automotive safety technology can't avoid collisions. With the development of automotive technology and the transformation of automotive safety technology concepts, the concept of active safety technology has gained popularity. The main effect of active safety technology is that the driver can control the car freely, with either of lateral or longitudinal movements as smooth as possible, being able to predict/avoid collision hazards, and ensure car safety in emergencies. Common active safety configurations include traction control system, electric brake force distribution, antilock brake system, electronic stability control, etc ${ }^{[1]}$.

With the rapid development of intelligent driving technology in recent years, automotive active safety technology has also come into a period of rapid development. Utilization of a series of new technologies such as collision warning, automatic emergency breaking and blind spot detection, greatly reduces the occurrence of car collision accidents

\footnotetext{
*Corresponding author: leibin@catarc.ac.cn
} 
and improves driving safety. Among them, automatic emergency braking technology plays an important role in avoiding collision accidents, and vehicle companies also attach great importance to the research and development of automatic emergency braking technology. The automatic emergency braking system is mainly composed of three major modules: control module, ranging module and braking module. However, due to the different automatic emergency braking control logic of different companies and the different basic braking capabilities of each model, the automatic emergency braking effect of different models also varies.

At present, most of the test research on automatic emergency braking system technology is focusing on the objective test category, while there are few related studies on automatic emergency braking subjective evaluation based on users' experiences. Therefore,this article through analyzing the principles and technical features of the automatic emergency braking system, selects vehicle-to-vehicle, vehicle-to-person and other typical scenarios for subjective evaluation of automatic emergency braking, and builds a subjective evaluation system, which can be used as a supplement to the objective test of automatic emergency braking, providing a new evaluation method for vehicle companies in the evaluation of automatic emergency braking system.

\section{Analysis on technical features of Automatic Emergency Braking}

\subsection{Definition of automatic emergency braking}

Automatic Emergency Braking (AEB), that is, real-time monitoring of the driving environment in front of the vehicle, automatically activates the vehicle braking system slowing down the vehicle to avoid collision or reduce collision effect. when the vehicle detects a safe distance from the vehicle in front or other road users or obstacles where collision may occur.

The AEB system architecture is mainly composed of three layers: perception layer, decision layer and execution layer. as shown in figure 1:

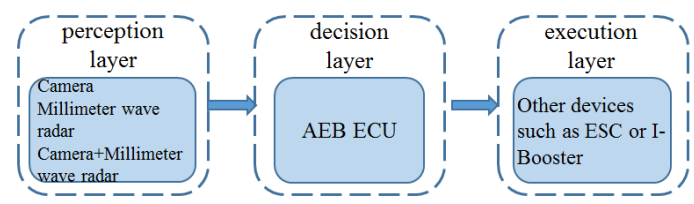

Fig. 1. AEB system architecture.

In the perception layer part, there are three common schemes: camera scheme, millimeter wave radar scheme, camera and millimeter wave radar fusion scheme. The current mainstream solution is the combination solution, which can combine the advantages of the two sensors thus distinguish the type of target even small targets through the camera, and detect the distance information through the radar, and then combine two kinds of signal to increase the system stability and reduce the false trigger rate.

The decision layer is the main control unit of the AEB system. After receiving the information sent by the radar or camera, it completes the signal processing, judges the relative position relationship with the target in front, evaluates the possibility of collision according to the internally set algorithm logic, and then send out the execute command.

The execution layer part is the emergency braking actuator, usually the ESC system, but there are other types of actuator, such as Bosch i-booster. 


\subsection{Automatic emergency braking work flow}

The main functions of the AEB system are Forward Collision Warning (FCW) and Automatic Braking (AB). The FCW monitors the driving environment in front of the vehicle in real time, and trigger an alarm to the driver when judging the possibility of a forward collision, with warning methods of sound, light, and tactile. This stage can be recognized as the early part of the AEB function; The automatic braking part $(\mathrm{AB})$ starts full brake deceleration when the system detects the danger of a collision, which stage can be recognized as the late part of the AEB function ${ }^{[2]}$. The work flow of the AEB system is shown in Figure 2:

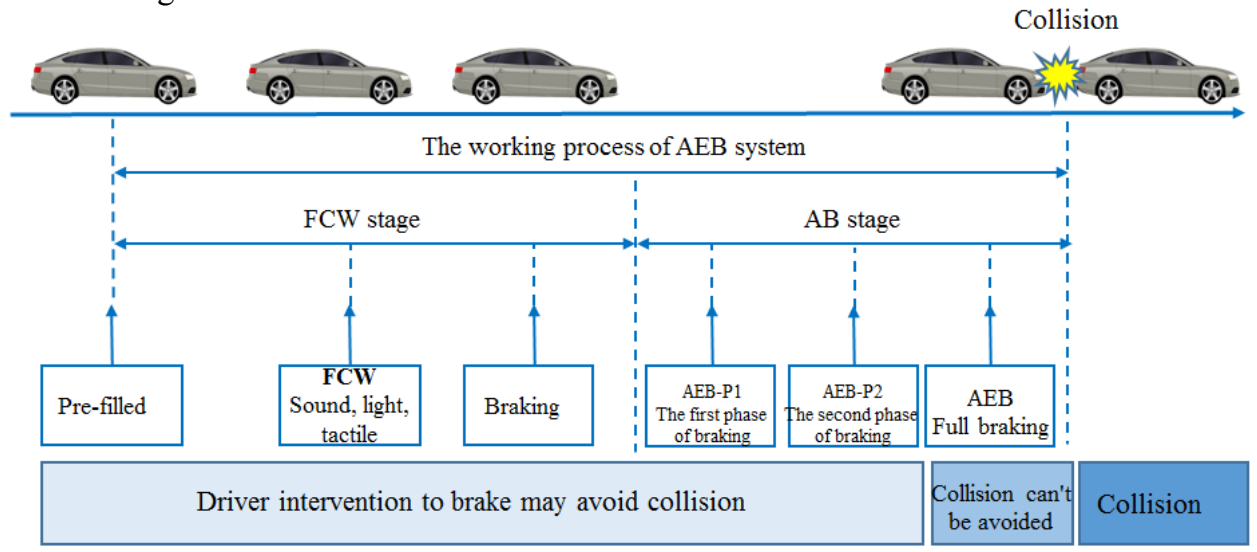

Fig. 2. AEB system work flow.

\subsection{Status of automatic emergency braking technology}

At the beginning of the development of AEB technology, there was only FCW function which would triggers the alarm when there's rear-end collision risk, by detecting the distance to the front vehicle by the millimeter wave radar and calculating the pre-collision time. With the development of sensor technology and ESC technology, the AEB function combining alarm and braking has gradually appeared. The current AEB technology can not only detect vehicles, but also detect pedestrians, bicycles, motorcycles and other vulnerable road users to avoid collisions.

\section{Constructing of subjective evaluation system for AEB}

Based on the technical features of automatic emergency braking and the typical scenarios of vehicle-to-vehicle and vehicle-to-person, this article structures a related subjective evaluation system.

\subsection{Subjective evaluation of automatic emergency braking}

Subjective evaluation of automatic emergency braking refers to that the trained assessors use body senses to evaluate the overall performance of automatic emergency braking in typical scenes per the evaluation criteria, and analyze, describe the evaluation results by quickly perceiving automatic emergency braking performance level. 


\subsection{Scoring basis}

Ten-point scoring method is recommended in order to make the subjective evaluation data reflecting the slight difference between vehicles' performances. 0.25 point is used as the minimum indexing value, that is, 1 point is divided into four score levels of $0,0.25,0.5$, and $0.75^{[3]}$. The basis for the ten-point scoring system is shown in Table 1:

Table 1. Subjective evaluation scoring basis.

\begin{tabular}{|c|c|c|c|c|}
\hline score & evaluation & category & evaluator & defect \\
\hline 1 & very poor & \multirow{4}{*}{ unacceptable } & \multirow{3}{*}{$\begin{array}{l}\text { all consumers } \\
\text { complain }\end{array}$} & loss of functions \\
\hline 2 & poor & & & serious defect \\
\hline 3 & relatively poor & & & defect \\
\hline 4 & slightly poor & & \multirow{2}{*}{$\begin{array}{c}\text { ordinary } \\
\text { consumers } \\
\text { complain }\end{array}$} & $\begin{array}{c}\text { need } \\
\text { improvement }\end{array}$ \\
\hline 5 & marginal & $\begin{array}{l}\text { conditionally } \\
\text { acceptable }\end{array}$ & & more \\
\hline 6 & acceptable & \multirow{5}{*}{ acceptable } & \multirow{2}{*}{$\begin{array}{c}\text { critical } \\
\text { consumers } \\
\text { complain }\end{array}$} & relatively less \\
\hline 7 & $\begin{array}{c}\text { relatively } \\
\text { good }\end{array}$ & & & less \\
\hline 8 & good & & \multirow{2}{*}{$\begin{array}{c}\text { trained } \\
\text { engineer } \\
\text { complain }\end{array}$} & extremely less \\
\hline 9 & very good & & & almost no feel \\
\hline 10 & perfect & & no complaint & no feel \\
\hline
\end{tabular}

\subsection{Evaluation scenarios}

This article mainly studies the functions of vehicle-to-vehicle and vehicle-to-vulnerable road users of AEB, for the following four typical scenes ${ }^{[4]}$ :

1) Vehicle-to-Vehicle

In this scenario, CCRs are evaluated (the test vehicle moves at a constant speed, while the target vehicle is stationary), and a soft balloon trolley is set as the target. The evaluation scenario is shown in Figure 3:

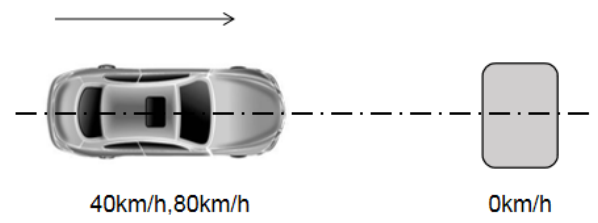

Fig. 3. CCRs evaluation scenario.

When evaluating CCRs, the test vehicle travel along the path of the target vehicle which is stationary. $40 \mathrm{~km} / \mathrm{h}$ speed is adopted to evaluate AEB function of the test vehicle, while $80 \mathrm{~km} / \mathrm{h}$ to evaluate $\mathrm{FCW}$ function.

2) Vehicle-to-Adult Pedestrian

This evaluation is for the scenario where the target in front is adult pedestrian. A stationary adult dummy is picked as the target. The evaluation scenario is shown in Figure 4: 


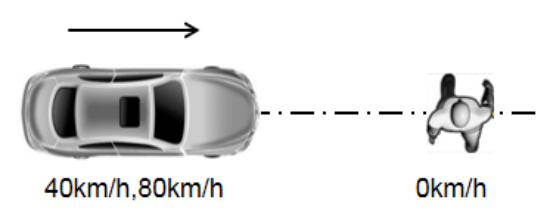

Fig. 4. Vehicle-to-adult pedestrian evaluation scenario.

In the scenario of vehicle-to-adult, the test vehicle and travel along the path of the adult pedestrian that is stationary. $40 \mathrm{~km} / \mathrm{h}$ speed is adopted to evaluate AEB function of the test vehicle, while $80 \mathrm{~km} / \mathrm{h}$ to evaluate $\mathrm{FCW}$ function.

3) Vehicle-to- Child Pedestrian

This evaluation is for the scenario where the target in front is child pedestrian. A stationary child dummy is picked as the target. The evaluation scenario is shown in Figure 5:

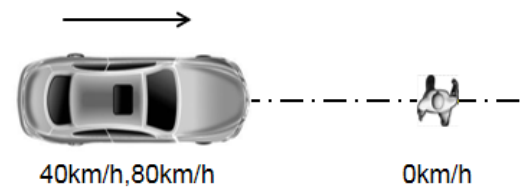

Fig. 5. Vehicle-to- child pedestrian evaluation scenario.

In the scenario of vehicle-to-child, the test vehicle and travel along the path of the child pedestrian that is stationary. $40 \mathrm{~km} / \mathrm{h}$ speed is adopted to evaluate AEB function of the test vehicle, while $80 \mathrm{~km} / \mathrm{h}$ to evaluate $\mathrm{FCW}$ function.

4) Vehicle-to- Bicycle

This evaluation is for the scenario where the target in front is bicycle. A stationary adult on bicycle dummy is picked as the target. The evaluation scenario is shown in Figure 6:

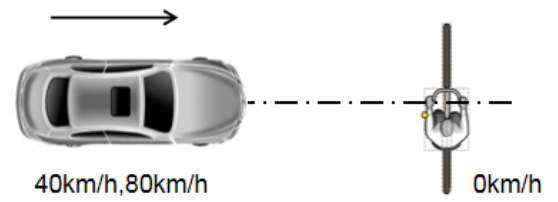

Fig. 6. Vehicle-to- bicycle valuation scenario.

In the scenario of vehicle-to- bicycle, he test vehicle and travel along the path of the bicycle which is stationary. $40 \mathrm{~km} / \mathrm{h}$ speed is adopted to evaluate AEB function of the test vehicle, while $80 \mathrm{~km} / \mathrm{h}$ to evaluate $\mathrm{FCW}$ function.

\subsection{Conditions of evaluation}

The subjective evaluation of AEB should meet the following conditions:

1) Personnel conditions:After being trained and passing the assessment, obtain the company's internal certificate;

2) Vehicle conditions: With necessary conditions of vehicles capable of meeting subjective evaluation requirements, the vehicle must have good dynamic performance, and no problem with the engine, chassis, body, electrical and electronic equipment, and automatic emergency braking systems;

3) Site: Clean, dry, straight concrete, asphalt or similar pavement; 
4) Weather: The average wind speed should be less than $5 \mathrm{~m} / \mathrm{s}$, sunny or cloudy;

5) Standards: Company internal subjective evaluation standard documents on automatic emergency braking.

\subsection{Subjective evaluation system}

This session evaluates the automatic emergency braking in three aspects: Human-computer Interaction, FCW function and AEB function, and introduces the subdivision items and subjective evaluation method:

\subsubsection{Items and methods of human-computer interaction subjective evaluation}

1) Icon visuality: to evaluate if the AEB icon indicator or signal is easy to recognize (including the icon size, the display location in the dash board, the color of the signal device, etc.)

2) System setting: Evaluate the operation logic and convenience of AEB system, switch logic and sensitivity setting, and whether the operation logic is clear and easy to understand, etc.

\subsubsection{Items and methods of FCW function subjective evaluation}

1) Warning method: To evaluate whether the warning method of the FCW system is reasonable, including whether the icon flashing, sound warning, and auxiliary alarm (seat belt pretension, HUD display, braking,etc.) warning method can attract the driver's attention and whether the warning intensity is appropriate.

2) Warning timing: With setting the warning sensitivity to medium or above medium, evaluate the gap between the warning time of FCW and the driver's psychological expectation, and whether the warning time is moderate.

3) False warning: To evaluate the ability of FCW system recognizing obstacles in front of the vehicle, whether there is false alarm, and the accuracy of obstacle recognition of the system.

\subsubsection{Items and methods of AEB function subjective evaluation}

1) Comfortableness: To evaluate the level of the braking deceleration, the pitch angle of the body, the sudden feeling and the attenuation of the pitch angle, the level of the working noise and so on when the AEB is engaged.

2) Efficiency: To evaluate whether the braking distance of the vehicle is moderate (neither of too far nor too close to the target is good) when the AEB is engaged, and the gap from the driver's expectation.

3) Sense of safety: To evaluate the driver's safety sense and confidence on the braking time and braking process when the AEB is engaged.

\section{Field test of subjective evaluation on Automatic Emergency Braking}

\subsection{The information of Vehicle AEB system}


Table 2. Vehicle Information.

\begin{tabular}{c|c|c}
\hline Vehicle & Sensor type & Technical features \\
\hline$\# 1$ & $\begin{array}{c}\text { Camera+Millimeter } \\
\text { wave radar }\end{array}$ & \multirow{2}{*}{ Identify vehicles and pedestrians } \\
\cline { 1 - 2 }$\# 2$ & $\begin{array}{c}\text { Camera+Millimeter } \\
\text { wave radar }\end{array}$ & \\
\hline$\# 3$ & Camera & \\
\hline
\end{tabular}

\subsection{Evaluation results of vehicle-to-vehicle}

The subjective evaluation results of the three vehicles in the vehicle-to-vehicle scenario are shown in Table 3:

Table 3. Subjective evaluation data of vehicle-to-vehicle.

\begin{tabular}{c|c|c|c|c}
\hline \multirow{2}{*}{ Item } & \multirow{2}{*}{ Index } & \multicolumn{3}{|c}{ Score } \\
\cline { 2 - 5 } & & $\# 1$ & $\# 2$ & $\# 3$ \\
\hline $\begin{array}{c}\text { Human-Computer } \\
\text { Interaction }\end{array}$ & Icon visuality & 7.0 & 5.5 & 5.5 \\
\cline { 2 - 5 } & System setting & 7.25 & 5.0 & 5.0 \\
\hline \multirow{3}{*}{ FCW function } & Warning method & 7.25 & 7.0 & 7.25 \\
\cline { 2 - 5 } & Warning timing & 7.0 & 6.75 & 7.25 \\
\cline { 2 - 5 } & False warning & 8.0 & 8.0 & 8.0 \\
\hline \multirow{2}{*}{$\begin{array}{c}\text { AEB } \\
\text { function }\end{array}$} & Comfortableness & 7.25 & 6.75 & 6.25 \\
\cline { 2 - 5 } & Efficiency & 7.0 & 6.75 & 7.5 \\
\cline { 2 - 5 } & Sense of safety & 7.0 & 6.5 & 7.5 \\
\hline
\end{tabular}

In the vehicle-to- vehicle scenario, all of three vehicles can successfully complete automatic emergency braking. In terms of human-computer interaction indicators, the AEB interfaces of \#2 and \#3 vehicles were set by the system default and can't be changed; In terms of the AEB comfortableness index, the \#3 braking is relatively sudden, and the comfortableness is not good.

\subsection{Evaluation results of vehicle-to- adult pedestrian}

The subjective evaluation results of the three vehicles in the vehicle-to- adult scenario are shown in Table 4:

Table 4. Subjective evaluation data of vehicle-to-adult.

\begin{tabular}{c|c|c|c|c}
\hline \multirow{2}{*}{ Item } & \multirow{2}{*}{ Index } & \multicolumn{3}{|c}{ Score } \\
\cline { 2 - 5 } & & $\# 1$ & $\# 2$ & $\# 3$ \\
\hline \multirow{2}{*}{$\begin{array}{c}\text { Human-Computer } \\
\text { Interaction }\end{array}$} & Icon visuality & 7.0 & 5.5 & 5.5 \\
\cline { 2 - 5 } & System setting & 7.25 & 5.0 & 5.0 \\
\hline \multirow{3}{*}{ FCW function } & Warning method & 7.25 & 1.0 & 7.25 \\
\cline { 2 - 5 } & Warning timing & 5.25 & 1.0 & 7.25 \\
\cline { 2 - 5 } & False warning & 8.0 & 1.0 & 8.0 \\
\hline \multirow{2}{*}{$\begin{array}{c}\text { AEB } \\
\text { function }\end{array}$} & Comfortableness & 7.25 & 1.0 & 6.25 \\
\cline { 2 - 5 } & Efficiency & 7.0 & 1.0 & 7.5 \\
\cline { 2 - 5 } & Sense of safety & 7.0 & 1.0 & 7.5 \\
\hline
\end{tabular}

In the vehicle-to-adult pedestrian scenario, the warning timing of the vehicle \#1 is close to the limit status; vehicle \#2 can't effectively identify the stationary adult dummy, and the AEB function was not activated. 


\subsection{Evaluation results of vehicle-to-child pedestrian}

The subjective evaluation results of the three vehicles in the vehicle-to-child scenario are shown in Table 5 :

Table 5. Subjective evaluation data of vehicle-to-child.

\begin{tabular}{c|c|c|c|c}
\hline \multirow{2}{*}{ Item } & \multirow{2}{*}{ Index } & \multicolumn{3}{|c}{ Score } \\
\cline { 2 - 4 } & & $\# 1$ & $\# 2$ & $\# 3$ \\
\hline \multirow{2}{*}{$\begin{array}{c}\text { Human-Computer } \\
\text { Interaction }\end{array}$} & Icon visuality & 7.0 & 5.5 & 5.5 \\
\cline { 2 - 5 } & System setting & 7.25 & 5.0 & 5.0 \\
\hline \multirow{3}{*}{ FCW function } & Warning method & 7.25 & 1.0 & 1.0 \\
\cline { 2 - 5 } & Warning timing & 5.25 & 1.0 & 1.0 \\
\cline { 2 - 5 } & False warning & 8.0 & 1.0 & 1.0 \\
\hline \multirow{2}{*}{$\begin{array}{c}\text { AEB } \\
\text { function }\end{array}$} & Comfortableness & 7.25 & 1.0 & 6.25 \\
\cline { 2 - 5 } & Efficiency & 7.0 & 1.0 & 7.5 \\
\cline { 2 - 5 } & Sense of safety & 7.0 & 1.0 & 7.5 \\
\hline
\end{tabular}

In the vehicle-to-child pedestrian scenario, the warning timing of the vehicle \#1 is close to the limit status; vehicle \#2 couldn't effectively identify the stationary child dummy, and the AEB function was not activated; vehicle \#3 was unable to recognize the stationary child dummy during high-speed driving and didn't output any warning.

\subsection{Evaluation results of vehicle-to-bicycle}

The subjective evaluation results of the three vehicles in the vehicle-to-bicycle scenario are shown in Table 6:

Table 6. Subjective evaluation data of vehicle-to-bicycle.

\begin{tabular}{c|c|c|c|c}
\hline \multirow{2}{*}{ Item } & \multirow{2}{*}{ Index } & \multicolumn{3}{|c}{ Score } \\
\cline { 2 - 4 } & & $\# 1$ & $\# 2$ & $\# 3$ \\
\hline \multirow{2}{*}{$\begin{array}{c}\text { Human-Computer } \\
\text { Interaction }\end{array}$} & Icon visuality & 7.0 & 5.5 & 5.5 \\
\cline { 2 - 5 } & System setting & 7.25 & 5.0 & 5.0 \\
\hline \multirow{3}{*}{\begin{tabular}{c} 
FCW function \\
\cline { 2 - 5 }
\end{tabular}} & Warning method & 1.0 & 1.0 & 1.0 \\
\cline { 2 - 5 } & Warning timing & 1.0 & 1.0 & 1.0 \\
\cline { 2 - 5 } & False arning & 1.0 & 1.0 & 1.0 \\
\hline \multirow{2}{*}{$\begin{array}{c}\text { AEB } \\
\text { function }\end{array}$} & Comfortableness & 1.0 & 1.0 & 6.25 \\
\cline { 2 - 5 } & Efficiency & 1.0 & 1.0 & 7.5 \\
\cline { 2 - 5 } & Sense of safety & 1.0 & 1.0 & 7.5 \\
\hline
\end{tabular}

In the vehicle-to-bicycle scenario, vehicles \#1 and \#2 couldn't effectively identify the stationary bicycle dummy, and the AEB function was not activated; vehicle \#3 was unable to recognize the stationary adult bicycle dummy during high-speed driving, and didn't output any warning.

\subsection{Comprehensive analysis results}

Through analyzing the scoring trend of the three indicators of human-computer interaction, FCW function and AEB function in automatic emergency braking, in four scenarios of vehicle-to-vehicle, vehicle-to- adult, vehicle-to-child, and vehicle-to-bicycle (average scores from Table 3-6), the technical maturity of the current automatic emergency braking in different scenarios can be verified as Figure 7: 


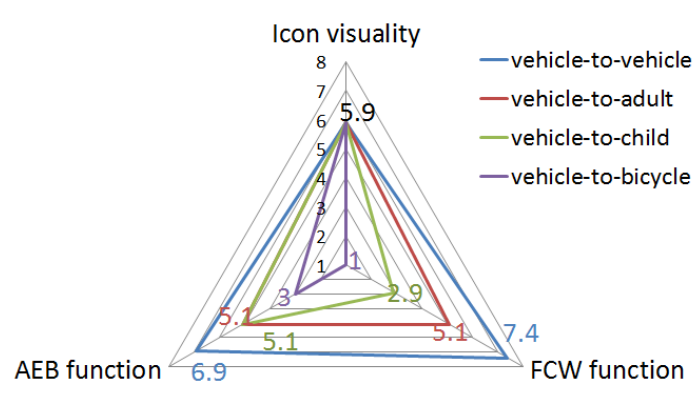

Fig. 7. Score comparison.

As shown in Figure 7, in the four typical scenarios of vehicle-to-vehicle, vehicle-to-adult, vehicle-to-child and vehicle-to-bicycle, they share the same score on human-computer interaction indicators, while AEB interfaces of some models were set by the system default and can't be changed that is not convenient for driver to set. The warning function and the automatic emergency braking function can't work successfully in the scenarios except for vehicle-to-vehicle scenario. Several systems are unable to accurately and effectively identify pedestrians or bicycles, resulting in no response from AEB system functions.

\section{Conclusion}

Through analyzing the technical features of automatic emergency braking, this article studied the vehicle field tests in four typical scenarios, and built an subjective evaluation system which is executable and can help on comprehensive, effective evaluation on the vehicle's automatic emergency braking at subjective evaluation aspect.

Through analyzing the evaluation results, it can be found that the current automatic emergency braking appears to be more mature in the scenario of vehicle-to-vehicle than other scenarios. In the scenarios of vehicle-to-adult, vehicle-to-child, and vehicle-tobicycle, some AEB systems can't effectively identify the target and result in the failure of the warning function or the automatic emergency braking function which proves that the current AEB function still has certain restrictions. The AEB systems equipped with multiple sensors (radars, cameras, etc.) can identify multiple targets as long as the design adopts combined algorithms of data processing and target classification with strengthening the recognizing capability of perception layer and expanding its effective target database.

With the development of millimeter-wave radar, cameras, laser radar and V2X technology, AEB technology will also go various with promoted performance, which will cover more complex scenarios such as intersections, vehicle turning, back-moving, higher speed targets, etc.

\section{References}

1. Chen Yinding,Chen Donggui.Technology and future development of automotive safety system [J]. Auto Time, 2019(18):159-160.

2. Hermann W,Stephan H,Felix L,Christina S.. Handbook of Driver Assistance Systems[J]. 2015.

3. Wu Lijun. Automobile Vehicle Performance Subjective Evaluation [M]. Beijing: Beijing Institute of Technology Press,2016. 
4. EUROPEAN NEW CAR ASSESSMENT PROGRAMME,TEST PROTOCOL-AEB VRU systems,Version 3.0.2 July2019[S/OL].https:// www. euroncap.com/en/. 